\title{
ON TOPOLOGIES OF MAXIMALLY ALMOST PERIODIC GROUPS
}

\author{
TER-JENQ HUANG
}

\begin{abstract}
It is proved that a necessary and sufficient condition for the group topology of any maximally almost periodic Hausdorff group to coincide with the weak topology of the group in which every complex-valued continuous almost periodic function on the group is continuous is that the group has the equivalent left and right uniform structures. The sufficiency of this condition generalizes the recent results of Glicksberg and Venkataraman concerning the group topology and the weak topology of an abelian Hausdorff group induced by the set of all continuous characters of the group.
\end{abstract}

1. Introduction. Let $G$ be a topological group. The weak topology of $G$ is defined to be the weakest topology of $G$ in which every complex-valued continuous almost periodic function on $G$ is continuous. $G$ is said to be maximally almost periodic (MAP) provided that the set of all complex-valued continuous almost periodic functions on $G$ separates the elements of $G$. For references, see, e.g., J. von Neumann [9], and J. Kelley [7]. Throughout this paper, all topological groups are assumed to be Hausdorff, and the weak topology of the group is always denoted by $w$. The purpose of this paper is to prove the following:

THEOREM. Let $(G, t)$ be a MAP group with the group topology $t$. Then the following conditions are equivalent:

(1) $t=w$;

(2) $G$ has the equivalent left and right uniform structures.

REMARK. There are locally compact groups which are MAP but the left and right uniform structures are not equivalent; see, e.g., Huang [5] and Murakami [8].

In [3], Glicksberg proved that if $G$ is a locally compact abelian group, then every subset of $G$ compact in the weak topology of $G$ induced by the set of all its continuous characters is also compact in the group topology. He proved also that if $t_{1}, t_{2}$ are two locally compact Hausdorff topologies on an abelian group $G$ which give rise to the same continuous characters, then $t_{1}=t_{2}$. Hughes [6] extended these results to any locally compact (not necessarily

Received by the editors April 25, 1977 and, in revised form, September 11, 1977.

AMS (MOS) subject classifications (1970). Primary 43A60, 43A40, 22A05, 22B05.

Key words and phrases. Almost periodic function, maximally almost periodic group, character of an abelian group, Pontryagin duality. 
abelian) group $G$ (he considered the continuous irreducible unitary representations of $G$ instead of the continuous characters of $G$ ). In [10], Venkataraman generalized Glickberg's results to the class of abelian (not necessarily locally compact) groups which satisfy Pontryagin duality with rather different proofs. As it will be seen in \$3, the Theorem we have here enables us to give a unified approach to both Glicksberg and Venkataraman's results.

2. Proof of the Theorem. We need the following

Definition. Let $G$ be a topological group. A subset $A$ of $G$ is said to be discretely left (right) syndetic provided that there is a finite subset $K$ of $G$ such that $G=A K(G=K A)$.

REMARK. In [1], Alfsen and Holm used the term "left (right) relatively dense" instead of the term "discretely left (right) syndetic". The notion of "left (right) syndetic" was originally due to W. H. Gottschalk and G. A. Hedlund (Topological dynamics, Amer. Math. Soc. Colloq. Publ., vol. 36, Amer. Math. Soc., Providence, R. I., 1955).

LEMMA. Let $G$ be a topological group such that every neighborhood $V$ of the identity element $e$ of $G$ is discretely left syndetic. Then $G$ has the equivalent left and right uniform structures.

Proof. It suffices to show that every left index (member in the left uniform structure) is also a right index. Let $\mathrm{Ne}$ denote the neighborhood system of the identity element $e$ of $G$ and let $U$ be in Ne. Then there is $V_{1}$ in $N e$ such that $V_{1}=V_{1}^{-1}, V_{1}^{3} \subseteq U$ and there is a finite subset $K$ of $G$ such that $G=V_{1} K$. Since $K$ is compact, there is $V$ in $N e$ such that for every $k$ in $K$, $k^{-1} V k$ is a subset of $V_{1}$. We want to show that if $x, y$ are in $G$ and if $x^{-1} y$ is in $V$, then $x y^{-1}$ is also in $U$. Since $x^{-1} y$ is in $V$ and $x$ is in $G=V_{1} K, y=x v$ for some $v$ in $V$ and $x=v_{1} k$ for some $k$ in $K$. Now

$$
x y^{-1}=v_{1} k\left(v_{1} k v\right)^{-1}=v_{1}\left(k v^{-1} k^{-1}\right) v_{1}^{-1},
$$

which is in $V_{1}^{3}$ and, therefore, is in $U$. The proof of the Lemma is completed.

Proof of THE THEOREM. Assume (1). We prove (2). In view of the Lemma, it suffices to show that every $V$ in $N e$ is discretely left syndetic. It is readily seen that for every $\varepsilon>0$ and for every complex-valued continuous almost periodic function $f$ on $G$, the set

$$
N(e, f ; \varepsilon) \equiv\{x \mid x \text { in } G \text { such that }|f(x)-f(e)|<\varepsilon\}
$$

contains a discretely left syndetic subset $A(\varepsilon)$ of $G$. Since $t=w, V$ is also open in $w$, and thus there are complex-valued continuous almost periodic functions $f_{1}, f_{2}, \ldots, f_{n}$ on $G$, and for some $\varepsilon>0$,

$$
\begin{aligned}
N\left(e, f_{1}, f_{2}, \ldots, f_{n} ; \varepsilon\right) & \\
& \equiv\left\{x \mid \text { in } G \text { such that }\left|f_{i}(x)-f_{i}(e)\right|<\varepsilon, i=1,2, \ldots, n\right\}
\end{aligned}
$$

is a subset of $V$. 


$$
\bigcap_{i=1}^{n} A_{i}(\varepsilon) \subseteq \bigcap_{i=1}^{n} N\left(e, f_{i} ; \varepsilon\right)=N\left(e, f_{1}, f_{2}, \ldots, f_{n} ; \varepsilon\right) .
$$

It is readily seen that $\bigcap_{i=1}^{n} A_{i}(\varepsilon)$ is a discretely left syndetic subset of $G$ (this is the topological group version of $11^{\circ}$ Theorem, p. 5, Almost periodic functions by A. S. Besicovitch, Dover, 1954). Therefore, $V$ is a discretely left syndetic subset of $G$. This proves that (1) implies (2).

Assume (2). We prove (1). For each complex-valued continuous almost periodic function $f$ on $G$, define $d_{f}(x, y)=|f(x)-f(y)|$ for all $x, y$ in $G$. Then it is readily seen that $d_{f}$ is a gauge (pseudometric) uniformly continuous in the equivalent (left and right) uniform structures on $G$, determined by the metric $d(a, b)=|a-b|$ in $E^{\prime}$ (the real line). Let $D$ be the family of all gauges uniformly continuous in the equivalent (left and right) uniform structures on $G$, determined by the metric $d(a, b)=|a-b|$ in $E^{\prime}$. Then, since $G$ is MAP, the family $D$, which contains all $d_{f}$ defined as above, is a separating family. By [2, Theorem 11.4, p. 203], $t=t(D)$ where $t(D)$ is the topology on $G$ induced by the family $D$. To show that $t=w$, it suffices to show that $w$ is finer than $t$, or equivalently, to show that $w$ is finer than $t(D)$. To do this, let $\varepsilon>0, x_{0}$ be in $G$ and $f$ be a complex-valued continuous almost periodic function on $G$. Then we have

$$
\begin{aligned}
& \left\{x \mid x \text { in } G \text { and } d_{f}\left(x, x_{0}\right)<\varepsilon\right\} \\
& \quad=\left\{x \mid x \text { in } G \text { such that }\left|f(x)-f\left(x_{0}\right)\right|<\varepsilon\right\} \\
& \quad \equiv N\left(x_{0}, f ; \varepsilon\right) .
\end{aligned}
$$

This shows that $w$ is finer than $t(D)$. This proves that (2) implies (1). The proof of the Theorem is completed.

3. Some corollaries. As some immediate consequences of the Theorem, we have

COROllaRY 3.1. Let $(G, t)$ be a compact group or an abelian MAP group. Then $t=w$.

Since every locally compact abelian group as well as every abelian group that satisfies the Pontryagin duality is a MAP group, and since every complex-valued continuous almost periodic function on any abelian group is a uniform limit of a sequence of continuous characters of the group (cf., e.g., [4, (33.26) $(g)$, p. 312]), it follows that the weak topology $w$ of the group coincides with the weakest topology of the group induced by the set of all its continuous characters, and therefore this corollary generalizes both a theorem of Glicksberg [3, Theorem 1.2, p. 269] and a theorem of Venkataraman [10, Theorem 1.1, p. 591].

Corollary 3.2. Let $t_{1}, t_{2}$ be two Hausdorff topologies on a group $G$ such that the topological groups $\left(G, t_{1}\right)$ and $\left(G, t_{2}\right)$ are MAP groups with the same set of complex-valued continuous almost periodic functions defined on them. Suppose 
further that both $\left(G, t_{1}\right)$ and $\left(G, t_{2}\right)$ have the equivalent left and right uniform structures. Then $t_{1}=t_{2}$.

It is easy to see that this corollary generalizes both a theorem of Glicksberg $[3$, Corollary 2.4, p. 271] and a theorem of Venkataraman [10, Theorem 1.2, p. 591].

REMARK. It should be noted here that Venkataraman proved his Theorem 1.1 first, and by making use of Theorem 1.1 and some properties of a topological group that satisfies Pontryagin duality, he then proved his Theorem 1.2.

The author is grateful to the referee for calling his attention to the paper by Alfsen and Holm, as well as the term "left (right) relatively dense" used in the paper.

\section{REFERENCES}

1. E. M. Alfsen and P. Holm, A note on compact representations and almost periodicity in topological groups, Math. Scand. 10 (1962), 127-136.

2. J. Dugundji, Topology, Allyn and Bacon, Boston, Mass., 1966.

3. I. Glicksberg, Uniform boundedness for groups, Canad. J. Math. 14 (1962), 269-276.

4. E. Hewitt and K. A. Ross, Abstract harmonic analysis, Vol. II, Springer-Verlag, Berlin, 1970.

5. T.-J. Huang, On equicontinuous transformation groups (to appear).

6. R. Hughes, Compactness in locally compact groups, Bull. Amer. Math. Soc. 79 (1973), $122-123$.

7. J. L. Kelley, General topology, Van Nostrand, Princeton, N. J., 1955.

8. S. Murakami, Remarks on the structure of maximally almost periodic groups, Osaka Math. J. 2 (1950), 119-129.

9. J. von Neumann, Almost periodic functions in a group, Trans. Amer. Math. Soc. 36 (1934), 445-492.

10. R. Venkataraman, Compactness in abelian topological groups, Pacific. J. Math. 57 (1975), 591-595.

Department of Mathematics, State University of New York, College at Cortland, CORTLAND, New York 13045 